\title{
A longitudinal study of auditory evoked field and language development in young children
}

Yuko Yoshimura ${ }^{1}$, Mitsuru Kikuchi ${ }^{1 *}$, Sanae Ueno ${ }^{1}$, Kiyomi Shitamichi², Gerard B. Remijn ${ }^{3}$,

Hirotoshi Hiraishi ${ }^{1}$, Chiaki Hasegawa ${ }^{1}, \mathrm{Naoki}_{\text {Furutani }}{ }^{2}$, Manabu Oi $^{1}$, Toshio Munesue ${ }^{1}$,

Tsunehisa Tsubokawa ${ }^{4}$, Haruhiro Higashida ${ }^{1}$ and Yoshio Minabe ${ }^{2}$

1) Research Center for Child Mental Development, Kanazawa University, Kanazawa, Japan

2) Department of Psychiatry and Neurobiology, Graduate School of Medical Science,

Kanazawa University, Kanazawa, Japan

3) International Education Center, Kyushu University, Fukuoka, Japan

4) Department of Anesthesiology, Graduate School of Medical Science, Kanazawa

University, Kanazawa, Japan

* Corresponding author. Research Center for Child Mental Development, Kanazawa

University, 13-1 Takara-machi, Kanazawa 920-8641, Japan

E-mail address: mitsuru@zc4.so-net.ne.jp, Tel: +81-76-265-2304 
Number of pages: 35 ,

Number of figures: 5 ; tables: 4,

Numbers of words: Abstract, 239; Introduction, 653; Discussion, 1,263 


\begin{abstract}
The relationship between language development in early childhood and the maturation of brain functions related to the human voice remains unclear. Because the development of the auditory system likely correlates with language development in young children, we investigated the relationship between the auditory evoked field (AEF) and language development using non-invasive child-customized magnetoencephalography (MEG) in a longitudinal design.
\end{abstract}

Twenty typically developing children were recruited (aged 36-75 months old at the first measurement). These children were re-investigated 11-25 months after the first measurement. The AEF component $\mathrm{P} 1 \mathrm{~m}$ was examined to investigate the developmental changes in each participant's neural brain response to vocal stimuli. In addition, we examined the relationships between brain responses and language performance. $\mathrm{P} 1 \mathrm{~m}$ peak amplitude in response to vocal stimuli significantly increased in both hemispheres in the second measurement compared to the first measurement. However, no differences were observed in P1m latency. Notably, our results reveal that children with greater increases in $\mathrm{P} 1 \mathrm{~m}$ amplitude in the left hemisphere performed better on linguistic tests. Thus, our results indicate that $\mathrm{P} 1 \mathrm{~m}$ evoked by vocal stimuli is a neurophysiological marker for language development in young children. Additionally, 
MEG is a technique that can be used to investigate the maturation of the auditory cortex based on auditory evoked fields in young children. This study is the first to demonstrate a significant relationship between the development of the auditory processing system and the development of language abilities in young children. 
Keywords: longitudinal study, magnetoencephalography (MEG), young children, auditory evoked field, language conceptual inference ability, human voice 


\section{Introduction}

Language acquisition in early childhood is one of the most fundamental human traits.

Dramatic developmental changes occur in the brains of young children in concert with

this ability (Sakai, 2005). Our previous magnetoencephalography (MEG) study reported

that the auditory evoked brain response (i.e., P50m) to vocal stimuli in the left

hemisphere was significantly correlated with language conceptual inference ability in

normal 2- to 5-year-old children (Yoshimura et al., 2012) and 3- to 7-year-old children

(Yoshimura et al., 2013). Although the relationships between longitudinal changes in

the brain response to vocal stimuli and language development remain unknown,

Chouldhury and Benasich (2011) examined auditory responses to tone stimuli using

electroencephalography (EEG) in longitudinal samples of typically developing children

and children at higher risk for language disorders between 6 and 48 months of age.

Their results demonstrated that infants with larger responses and shorter peak

latencies from 6 to 9 months old had better language and cognitive skills at 3 and 4

years old. Moreover, other studies have reported that brain responses to auditory

stimuli in infancy and childhood are associated with subsequent language-related skills

(Espy et al., 2004) or language impairments such as dyslexia (Leppänen et al., 2012;

Leppänen et al., 2010). However, to date, there has been no study that has followed this 
change in the brain response evoked by vocal stimuli using a longitudinal design or that has investigated its relationship to language development in young, typically developing children without family risk factors for language-related impairment (e.g., dyslexia).

The purpose of this study was to investigate the relationship between longitudinal changes in the auditory evoked response $(\mathrm{P} 1 \mathrm{~m})$ to vocal stimuli and language development in early childhood in typically developing children using MEG. A previous study demonstrated that the maturational patterns in auditory processing differ depending on the type of stimulus (e.g., speech or non-speech stimuli) (Pang and Taylor, 2000). In the present study, we focused on human voice-evoked responses. Understanding the normal maturation pattern of AEFs evoked by the human voice may aid in the development of neurophysiological techniques for evaluating the central auditory maturation that coincides with language development in young children.

$\mathrm{P} 1(\mathrm{~m})$ is a prominent component in $1^{-}$to $10^{-}$year-old children (Gilley et al., 2005; Oram Cardy et al., 2004; Ponton et al., 2002; Sharma et al., 1997) and provides insight into the development of auditory processing. P1m is thought to be a suitable metric for measuring changes in auditory input for speech-like signals (Chait et al., 2004; Hertrich et al., 2000). A recent study indicated that $\mathrm{P} 1 \mathrm{~m}$ is sensitive to the place-of-articulation 
features of speech and their co-articulatory processes (Tavabi et al., 2007). In previous MEG studies, this component has been alternatively labeled M50 (Oram Cardy et al., 2004) or P100m (Orekhova et al., 2013; Orekhova et al., 2012). In our previous study, this component was labeled P50m (Yoshimura et al., 2012). Some EEG studies have also labeled this component P1 (Gilley et al., 2005; Ponton et al., 2002). According to Orekhova et al. (2012), the P1m component at approximately $100 \mathrm{~ms}$ after stimulation is the most prominent component of the auditory evoked magnetic field response in children, and the equivalent current dipoles (ECD) of $\mathrm{P} 1 \mathrm{~m}$ have a predominantly anterosuperior direction. This component can be reliably identified in the majority of children because of its large amplitude (Oram Cardy et al., 2004). This component, which we analyzed in our previous study, was identical to the results of previous MEG studies (Oram Cardy et al., 2004; Orekhova et al., 2012; Pihko et al., 2007). To avoid confusion, we call this component $\mathrm{P} 1 \mathrm{~m}$ in the present study.

We hypothesize that changes in P1m amplitude/latency are correlated with language development in early childhood. The aim of this study was to investigate the brain response to human vocal stimuli measured using a longitudinal design in $3^{-}$to 7-year-old typically developing children and to investigate the relationship between language development and the changes in brain response. 


\section{Methods}

\subsection{Participants}

Twenty children (3 females and 17 males) participated and were 36-75 months old at the first measurement. All children participated in additional measurements at $11^{-}$to 25-month intervals for 3 years. All participants were native Japanese and had no previous developmental, learning, or behavioral problems according to information obtained from their caregivers by questionnaire. All participants confirmed (through an interview with their caregivers) that they had no diagnosed hearing problems (mass screening at age 3) and that there were no hearing problems in their daily lives. Left- or right-hand dominance was determined based on the participants' preferences when handling objects, and all children were right-handed. All children participated in cognitive tasks and MEG measurements separately over 2 days. On the first day, the participants performed cognitive tests and were introduced to the environment used for the MEG measurements. The actual MEG measurements were performed on the second day. The caregivers consented to their child's participation in the study with full knowledge of the experimental nature of the research. Written informed consent was obtained from the caregivers prior to participation in this study. The Ethics Committee 
of Kanazawa University Hospital approved the methods and procedures, all of which were performed in accordance with the Declaration of Helsinki. The demographic data for all participants are presented in Table 1.

\subsection{Cognitive and language performance measurements}

The children were assessed using the Japanese adaptation of the Kaufman Assessment Battery for Children (K-ABC) (Kaufman and Kaufman, 1983), which is typically used to assess the cognitive skills of $30^{-}$to $155^{-}$-month-old children. To confirm the standardized scores of the mental processing and achievement scales in children, subtests from this battery that are appropriate for the ages of the children were used. In this study, the potential correlation between one of the components of the AEF (i.e., P1m amplitude and latency) and performance on a single language-related task (i.e., a subtest of K-ABC 'riddles') in a previous study (Yoshimura et al., 2012) was assessed. In the riddle task, children must respond to the examiner's question, such as "Which fruit has a rounded shape with a depression at the top where the stem is attached? The color of the skin can be red, green, yellow, or a combination of these colors." In this case, the answer is "an apple." The riddle task consists of 32 questions, and the questions are presented in ascending order of difficulty. The linguistic level is defined by the child's 
degree of achievement. The K-ABC 'riddles' subtest reflects conceptual language inference abilities (Kaufman and Kaufman, 1983).

\subsection{Magnetoencephalography recordings}

The conditions in the first and second MEG recordings were completely identical to those detailed in our previous study (Yoshimura et al., 2012). MEG data were recorded using a 151-channel SQUID (Superconducting Quantum Interference Device), whole-head coaxial gradiometer MEG system for children (PQ 1151R; Yokogawa/KIT, Kanazawa, Japan) in a magnetically shielded room (Daido Steel, Nagoya, Japan) installed at the MEG Center of Yokogawa Electric Corporation (Kanazawa, Japan). The custom child-sized MEG system facilitates the measurement of brain responses in young children, which would otherwise be difficult using conventional adult-sized MEG systems. The child MEG system ensures that sensors are easily and effectively

positioned for the child's brain and that head movements are constrained (Johnson et al., 2010). We determined the position of the head within the helmet by measuring the magnetic fields after passing currents through coils attached at 3 locations on the surface of the head, which served as fiduciary points relative to specific landmarks (the bilateral mastoid processes and nasion). Although we could not account for the effect of 
individual head shape on the accuracy of dipole estimation, to calculate ECD without magnetic resonance imaging anatomical data, a sphere (as a spherical model of the volume) conductor was fitted to the center of the helmet after confirming that each participant's head was located in the center of the MEG helmet by measuring the above-described three locations on the surface of the head (Yoshimura et al., 2012). An examiner remained in the room to encourage the children and to prevent movement throughout the analysis. Stimuli were presented while the child was in a supine position on the bed and viewed silent video programs projected onto a screen.

\subsection{Auditory evoked field stimuli and procedures}

The stimuli and procedure were based on our previous study (Yoshimura et al., 2012). MEG recordings were obtained from all participants during auditory syllable sound stimulation consisting of the Japanese syllable /ne/ (Yoshimura et al., 2012). We employed this syllable because /ne/ is one of the Japanese final sentence particles, which convey prosodic information (Anderson et al., 2007; Cook, 1990). The syllable /ne/ is often used in Japanese mother-child conversations and expresses a speaker's request for acknowledgement or empathy from the listener (Kajikawa et al., 2004; Squires, 2009). In the present study, we used typical oddball sequences consisting of standard 
stimuli (456 times, 83\%) and deviant stimuli (90 times, 17\%). In the standard stimulus, /ne/ was pronounced with a steady pitch contour, whereas in the deviant condition, /ne/ was pronounced with a falling pitch. Eventually, we adopted only standard stimuli for subsequent ECD estimations because a sufficient number of periods to calculate ECD remained after artifact rejection in all children. A female native Japanese speaker produced the /ne/ sounds, which were recorded using a condenser microphone (NT1-A; Rode, Silverwater, NSW, Australia) and a personal computer. The duration of the stimulus was $342 \mathrm{~ms}$, and the duration of the consonant/n/ was $65 \mathrm{~ms}$. In this study, the beginning of the vowel sound /e/ was defined as the onset time. The interstimulus interval (ISI) was $818 \mathrm{~ms}$. Both stimuli had an intensity level of approximately $65 \mathrm{~dB}$ (A-weighted) at the head position against a background noise of $43 \mathrm{~dB}$. Intensity was measured using an integrating sound level meter (LY20; Yokogawa, Tokyo, Japan). The stimulus was presented to participants binaurally through a hole in the MEG chamber using speakers (HK195 Speakers; Harman Kardon, Stamford, CT) placed outside the shielded room. The speaker was placed facing the participant's head from the direction of the foot, and the distance between the speaker and MEG helmet was $3.3 \mathrm{~m}$. The recording was 12 minutes long. 


\subsection{AEF acquisition and analysis}

The procedures for the AEF acquisition and analysis were identical to those in our previous study (Yoshimura et al., 2012). The bandpass-filtered MEG data $(0.16-200$ $\mathrm{Hz}$ ) were collected at a sampling rate of $1000 \mathrm{~Hz}$. The time series from the onset of the syllable stimulus at - 150 to $1000 \mathrm{~ms}$ and subsequent segments (at least 300 for standard stimuli) were averaged for each sensor after baseline correction (-50 to $0 \mathrm{~ms})$. Segments contaminated with artifacts (eye-blink and eye and body movements, typically more than $\pm 4 \mathrm{pT}$ ) were excluded from the analysis. A single ECD model was used to estimate current sources in the activated cerebral cortex using more than 30 sensors for each hemisphere (left and right) (Elberling et al., 1982). MegLaboratory 160 software (Yokogawa/KIT, Kanazawa, Japan) was used to estimate the localization of the current sources.

To identify P1m, we accepted estimated ECDs when (i) the goodness of fit (GOF) exceeded 80\%; (ii) the location of estimated dipoles using a single ECD model was stabilized within $\pm 5 \mathrm{~mm}$ of each coordinate for at least $6 \mathrm{~ms}$ during the P1m response; (iii) the dipole amplitudes were $\leq 80 \mathrm{nAm}$; and (iv) ECDs predominantly had an anterosuperior direction. The latency time point was defined as the maximum estimated dipole amplitude value obtained in accordance with the above criteria within 
a time window of 40 to $150 \mathrm{~ms}$. Regarding the $\mathrm{P} 1 \mathrm{~m}$ component coordinates, the center of a sphere as a spherical model of the volume conductor for the ECD estimation was defined as the origin, and the $\mathrm{x}^{-}, \mathrm{y}^{-}$, and $\mathrm{z}^{-}$coordinates represented the leftward direction, occipital direction, and vertex, respectively.

\subsection{Data analysis}

Each physiological variable of $\mathrm{P} 1 \mathrm{~m}$ (amplitude and latency) was analyzed using a two-way repeated measures analysis of variance (ANOVA). The two within-subjects variables were Measurement time (first vs. second measurement) and Hemisphere (left vs. right). Significance was established at $P<0.05$.

To compare the dipole source location between the first and second measurements, we employed a principal component analysis (PCA) because brain activations were not necessary along one of the Cartesian coordinates (i.e., $\mathrm{x}^{-}, \mathrm{y}^{-}$, and $\mathrm{z}^{-}$coordinates) of the source space. PCA identifies a coordinate system whose coordinates follow the most prominent spread of the data distribution. PCA allows us to replace the 3D statistical analysis of the locations with a $1 \mathrm{D}$ analysis of the representative coordinates for each hemisphere (Herrmann et al., 2011). The location (i.e., principal component score) differences for each hemisphere were compared between the first and second 
measurements using a paired $t$-test.

Pearson correlation coefficients were used to investigate the relationships between maturational changes in P1m (amplitude and latency) and language development. For the maturational change in the $\mathrm{P} 1 \mathrm{~m}$ amplitude variable, the values from the second measurement were divided by the values from the first measurement. For the maturational change in the $\mathrm{P} 1 \mathrm{~m}$ latency variable, the value was determined by subtracting the first measurement value from the second measurement value. The percent change in the standardized scores of the riddles in the $\mathrm{K}-\mathrm{ABC}$ was used as the developmental change in the language variables. Statistical significance was defined as $P<0.05$.

To evaluate possible confounding effects (i.e., age at the first measurement time, the time interval between the two measurements, and change in total cognitive ability) on the relationship between the change in P1m (amplitude and latency) and language development, we employed a multiple linear regression analysis to predict the change in P1m (i.e., dependent variable) using age at first measurement, the time interval between the two measurements, change in total cognitive ability and change in language ability as predictors (i.e., four independent variables). Statistical significance was defined as $P<0.05$. 


\section{Results}

\subsection{P1m amplitude and latency (Figures 1, 2, and 3)}

We were able to detect $\mathrm{P} 1 \mathrm{~m}$ at both measurement times (i.e., first and second measurements) in the right and left hemispheres of $17 / 20$ and $18 / 20$ participants, respectively. Figure 1 shows the typical auditory evoked fields and typical P1m component in one participant. Figure 2 shows that the $\mathrm{P} 1 \mathrm{~m}$ amplitude tends to increase with age, whereas the P1m latency tends to decrease with age (Figure 3) in both hemispheres.

\subsection{The changes in P1m amplitude and latency (Table 2)}

In $80 \%(16 / 20)$ of the participants, we were able to detect $\mathrm{P} 1 \mathrm{~m}$ in both hemispheres in both measurements. Therefore, those 16 participants were analyzed with a repeated measures two-way ANOVA. For the P1m amplitude, the repeated measures ANOVA indicated a main effect of Measurement time $(\mathrm{F}(1,15)=12.724, P=0.003)$ and Hemisphere $(\mathrm{F}(1,15)=18.935, P=0.001)$. However, there was no significant interaction (Measurement time $\times$ Hemisphere $)(\mathrm{F}(1,15)=4.414, P=0.053)$. These results indicate that the P1m amplitude was significantly higher in the left hemisphere compared to the 
right hemisphere, and the $\mathrm{P} 1 \mathrm{~m}$ amplitude was significantly higher in the second measurement compared to the first measurement, regardless of the hemisphere. Although there was no significant interaction, there was a trend suggesting that the increase in $\mathrm{P} 1 \mathrm{~m}$ amplitude in the left hemisphere between the first and second measurements was larger than that in the right hemisphere (left: first measurement, mean $( \pm$ standard deviation $)=18.3( \pm 7.2)$, second measurement, mean $( \pm$ standard

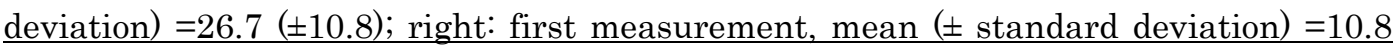

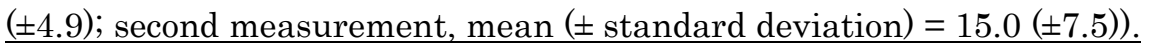

For the P1m latency, the repeated measures ANOVA indicated no significant main effect of Measurement time $(\mathrm{F}(1,15)=2.243, P=0.155)$ or Hemisphere $(\mathrm{F}(1,15)=4.192$, $P=0.059)$. There was no significant interaction (Measurement time $\times$ Hemisphere) $(\mathrm{F}$ $(1,15)=0.466, P=0.505)$. Although there was no significant main effect, we observed that $\mathrm{P} 1 \mathrm{~m}$ latency in the right hemisphere tended to be faster than in the left hemisphere for both measurements, and P1m latency tended to be faster for the second measurement than the first measurement in both hemispheres (first measurement: left, mean $( \pm$ standard deviation $)=89.0( \pm 22.4)$, right, mean $( \pm$ standard deviation $)=85.0$

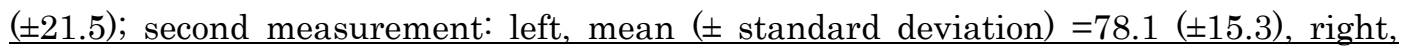
$\underline{\text { mean }}( \pm$ standard deviation $)=75.5( \pm 12.6))$. 


\subsection{The change in location of the estimated $\mathrm{P} 1 \mathrm{~m}$ dipole sources (Table 3)}

We were able to detect $\mathrm{P} 1 \mathrm{~m}$ for both measurements (i.e., first and second measurements) in the left and right hemispheres of $17 / 20$ and $18 / 20$ participants, respectively. Therefore, in conjunction with these two measurements, 34 and 36 dipole locations were used for the PCA of the left and right hemispheres, respectively. The PCA indicated that in both hemispheres, one component (i.e., coordinate) had an eigenvalue greater than one. In both hemispheres, positive values of the first component (coordinate) occasionally corresponded to the medial-vertex direction. A significant difference was observed for the PCA score of the right hemisphere between the first and second measurements ( $\mathrm{df}=17, \mathrm{t}=-3.58, P=0.002)$ (Table 3$)$. This result indicates that the location of the $\mathrm{P} 1 \mathrm{~m}$ dipole source in the right hemisphere shifted toward the medial-vertex direction between the first and second measurements. In the left hemisphere, there was no significant difference between the first and second measurements $(\mathrm{df}=16, \mathrm{t}=0.31, P>0.05)($ Table 3$)$.

\subsection{The relationships between changes in $\mathrm{P} 1 \mathrm{~m}$ amplitude and latency and language}

development (Figures 4 and 5) 
We detected P1m for both measurements (i.e., first and second measurements) in the right and left hemispheres of 17/20 and 18/20 participants, respectively. Therefore, 17 and 18 participants were used to examine the right and left hemispheres, respectively, in the correlation analysis for longitudinal changes. A significant positive correlation was observed between the $\mathrm{P} 1 \mathrm{~m}$ amplitude change in the left hemisphere and the percent change in the language conceptual inference task (i.e., riddles) $(n=17, r=0.526$, $P=0.028)$. However, this correlation was not significant for the right hemisphere $(\mathrm{n}=$ $18, \mathrm{r}=0.088, P>0.05$ ) (Figure 4). The change in $\mathrm{P} 1 \mathrm{~m}$ latency was not significantly correlated with the percent change in the language conceptual inference task (i.e., riddles) for either hemisphere (Figure 5).

In the multiple regression model shown in Table 4, the time interval between the two measurements $(\mathrm{n}=17, \beta=0.476, P=0.030)$ and the percent change in the language conceptual inference task $(\mathrm{n}=17, B=0.473, P=0.027)$ were significant predictors of the P1m amplitude change in the left hemisphere. By contrast, age at the first measurement ( $\mathrm{n}=17, \beta=0.027, P>0.05)$ and change in total cognitive ability (i.e., change in the mental processing scale according to the $\mathrm{K}-\mathrm{ABC})(\mathrm{n}=17, B=0.277, P>$ 0.05) showed no significant effects. In the right hemisphere $(n=18)$, the coefficient of multiple determination for multiple regression (i.e., $\mathrm{R}^{2}=0.260$ ) was not significant $(P=$ 
0.380), and no independent variables reached statistical significance, as indicated by the multiple linear regression (Table 4). For the change in $\mathrm{P} 1 \mathrm{~m}$ latency, no variables reached statistical significance for either hemisphere, as indicated by the multiple linear regression. There was no significant correlation among independent variables $(\mathrm{r}$ values ranged from -0.407 to 0.171 ). The correlation coefficients between independent variables were as follows: 'change in language conceptual inference tasks' and 'change in mental processing scale' $(r=0.171)$, 'change in language conceptual inference tasks' and 'age at first measurement' $(r=-0.126)$, 'change in language conceptual inference $\underline{\text { tasks' and 'time interval' }(r=0.001) \text {, 'change in mental processing scale' and 'age at first }}$ measurement' $(r=0.112)$, 'change in mental processing scale' and 'time interval' $(r=$

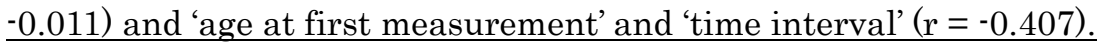

\section{Discussion}

This study demonstrated a relationship between longitudinal change in P1m elicited by vocal stimuli and language development in young children. Our results indicate that the maturation of the auditory cortex in the left hemisphere is associated with language development. In this study, we also demonstrated that our child-customized MEG device allowed us to acquire bilateral (i.e., left and right hemispheres) simultaneous 
brain responses in conscious young children. MEG is currently a suitable technology for measuring the absolute value of brain auditory responses in young children. Because it shows a good signal-to-noise ratio, excellent temporal resolution, and a long history of EEG studies (to aid study interpretations), is safe and non-invasive, and is supported by recent technical advancements (e.g., child custom-sized MEG), MEG is an excellent tool for investigating the spatio-temporal dynamics of neural activation associated with sensory perception in early childhood (Huotilainen et al., 2008; Pang, 2011).

The component analyzed in our previous study was identical to P100m, M50 and P1m responses, which have been demonstrated in previous MEG studies (Oram Cardy et al., 2004; Orekhova et al., 2012; Pihko et al., 2007). To avoid confusion, we called this component $\mathrm{P} 1 \mathrm{~m}$ in the present study.

We noted a significant relationship between language development and increased P1m amplitude in the left hemisphere. This result supports our previous cross-sectional finding that $\mathrm{P} 1 \mathrm{~m}$ amplitude in the left hemisphere is significantly correlated with language conceptual ability in 2- to- 5-year-old children (Yoshimura et al., 2012). In terms of the relationship between the auditory brain response and language development, Oram Cardy et al. (2008) also reported that M50 latency to tone stimuli in the right hemisphere is a predictor of language impairment (i.e., specific language 
impairment (SLI) and autism spectrum disorder) in 7- to 18-year-old children and adolescents (Oram Cardy et al., 2008). In addition, Chouldhury and Benasich (2011) demonstrated that significant age-related changes in the latency and amplitude of P1 and later components in response to tone stimuli (70-ms ISI) predicted language abilities at 36 and 48 months of age. However, in typically developing children, the relationships between longitudinal language development and earlier components (e.g., P1m) have remained controversial. Our child-customized MEG system allows us to provide new evidence that developmental changes in $\mathrm{P} 1 \mathrm{~m}$ amplitude in response to vocal stimuli reflect the maturation of language-related brain regions.

From the first to the second measurement, we observed a significant increase in $\mathrm{P} 1 \mathrm{~m}$ amplitude in both hemispheres. Furthermore, P1m amplitude in the left hemisphere was significantly larger than in the right hemisphere at both measurements. This $\mathrm{P} 1 \mathrm{~m}$ left hemispheric dominance is consistent with other AEF studies (Fujioka et al., 2006; Heim et al., 2003; Tavabi et al., 2007; Yoshimura et al., 2012). However, no previous study has examined longitudinal change in the auditory evoked field (i.e., P1m) to vocal stimuli. Only Fujioka et al. (2006) has examined changes in P1m amplitude to non-vocal sounds (i.e., noise burst and violin tones) in 4- to 6-year-old typically developing children. These authors investigated the effects of 1 year of music lessons on the P100m 
(P1m) component. Over four repeated MEG measurements, a significantly greater response to violin sounds in the left hemisphere compared to the right hemisphere was observed. In our results, left-hemispheric dominance in P1m was enhanced for vocal sounds. In our study, the participants did not receive structured intervention (e.g., musical training) during the interval; however, these participants would have been affected by language input and would have had daily exposure to language from their parents and other children. We speculate that the same brain development mechanisms may be associated with the perception of both the violin and human voice. The identical developmental changes in the auditory cortex in the left hemisphere were observed after continuous exposure to violin sound and human voice. Pihko et al. (2007) also reported an effect of language intervention on P1m amplitude and latency for children with SLI. The authors observed that after language training, P1m amplitudes increased in response to auditory stimuli, although this result was not significant (Pihko et al., 2007).

It appears that $\mathrm{P} 1 \mathrm{~m}$ amplitude is a dynamic factor that is influenced by longitudinal auditory input. However, in this study, we investigated P1m only using one type of auditory stimulus (i.e., the human voice /ne/). Therefore, we cannot determine whether our results are specific to human vocal stimuli. 
Increases in $\mathrm{P} 1$ amplitude with age have been reported in several previous developmental studies (Gilley et al., 2005; Ponton et al., 2002; Sussman et al., 2008), as was observed in our study. Sussman et al. (2008) revealed that P1 amplitudes were larger for 8-year-old children than for older age groups with short ISI (i.e., $<1$ s). Gilley et al. (2005) reported that the increase in the P1 amplitude was not significant for three groups (3-4 years old, 5-6 years old and 7-8 years old); however, there was a tendency for $\mathrm{P} 1$ amplitudes to increase with age until approximately $7-8$ years of age. Ponton et al. (2002) also reported that P1 peak amplitudes increased from 5-6 to 10 years of age. These results are consistent with our findings, in which $\mathrm{P} 1 \mathrm{~m}$ amplitudes increased from 3 to 7 years of age. Using a longitudinal design, our results provide new evidence in young children (including 3-4-year-olds).

In the present study, we did not observe a significant P1m latency change in either hemisphere within our 11-25 month interval, although we observed a tendency toward decline (Figure 3). Previous cross-sectional studies in children older than 5 have reported a change in P1m latency with age (Cunningham et al., 2000; Fujioka et al., 2006; Kotilahti et al., 2010; Ponton et al., 2002). Cunningham et al. (2000) and Ponton et al. (2002) reported that $\mathrm{P} 1$ latencies in response to brief click trains decreased with age; however, these changes were not significant during early school-age years (i.e., 
from 5 to 12 years old). Significant changes appear to occur later in childhood, as these authors also reported that P1 latencies in children older than 13 were significantly shorter than those of 5- to 10-year-old children. In addition, in a previous study with a longitudinal design, no significant decrease in P100m (P1m) latency was observed in a cross-section of 4- to 6-year-old children (Fujioka et al., 2006), consistent with our result.

Our results indicated that the location of the $\mathrm{P} 1 \mathrm{~m}$ dipole source in the right hemisphere shifted in the medial-vertex direction between the first and second measurements. Ponton et al. (2002) also reported a lateral-to-medial shift in the P1m source location and suggested that this source location shift is only observed in P1m (Ponton et al., 2002). Our results partially support this previous result for the right hemisphere (i.e., the location of $\mathrm{P} 1 \mathrm{~m}$ shifted medially).

The present study had some general limitations. First, we cannot discuss the detailed location of the magnetic field source as would be possible with anatomical images such as MRI. Future studies using child-friendly, open-type MRI devices and/or brain models that match the child brain (Fonov et al., 2011; Wilke et al., 2008) are necessary to reduce the uncertainties in the source level estimation. Second, the nominally lower correlation between $\mathrm{P} 1 \mathrm{~m}$ amplitude in the right hemisphere and 
language performance may be explained by the lower amplitude of $\mathrm{P} 1 \mathrm{~m}$ in the right hemisphere (i.e., lower signal/noise ratio). Further studies with larger sample sizes may reveal significant relationships for both hemispheres. Despite some limitations, we demonstrate a relationship between language development and longitudinal changes in $\mathrm{P} 1 \mathrm{~m}$. Our results revealed that $\mathrm{P} 1 \mathrm{~m}$ reflects the maturation of brain functions related to language development.

\section{Abbreviations}

AEF, auditory evoked field; ECD, equivalent current dipole; EEG, electroencephalography; ISI, interstimulus interval; K-ABC, Kaufman Assessment Battery for Children; MEG, magnetoencephalography; ANOVA, analyzed using a two-way repeated measures analysis of variance; PCA, principal component analysis;

SLI, specific language impairment.

\section{Acknowledgements}

This study was supported by a Grant-in-Aid for Specially Promoted Research (Research Number 24000012), the Center of Innovation Program from Japan Science and Technology Agency, JST, the Strategic Research Program for Brain Sciences from 
MEXT and a Grant-in-Aid for Young Scientists (B) (Grant Number 24791206), JAPAN.

\section{References}

Anderson, V., Hiramoto, M., Wong, A., 2007. Prosodic Analysis of the Interactional Particle Ne in Japanese Gendered Speech. Japanese/Korean Linguistics 15, 43-54.

Chait, M., Simon, J.Z., Poeppel, D., 2004. Auditory M50 and M100 responses to broadband noise: functional implications. Neuroreport 15, 2455-2458.

Cook, H.M., 1990. The sentence-final particle ne as a tool for cooperation in Japanese conversation. The Stanford Linguistic Association, Stanford.

Cunningham, J., Nicol, T., Zecker, S., Kraus, N., 2000. Speech-evoked neurophysiologic responses in children with learning problems: development and behavioral correlates of perception. Ear and Hearing 21, 554-568.

Elberling, C., Bak, C., Kofoed, B., Lebech, J., Saermark, K., 1982. Auditory magnetic fields from the human cerebral cortex: location and strength of an equivalent current dipole. cta Neurol Scand 65, 553-569.

Espy, K.A., Molfese, D.L., Molfese, V.J., Modglin, A., 2004. Development of auditory event-related potentials in young children and relations to word-level reading abilities at 
age 8 years. Annals of dyslexia 54, 9-38.

Fonov, V., Evans, A.C., Botteron, K., Almli, C.R., McKinstry, R.C., Collins, D.L., Brain Development Cooperative, G., 2011. Unbiased average age-appropriate atlases for pediatric studies. Neuroimage 54, 313-327.

Fujioka, T., Ross, B., Kakigi, R., Pantev, C., Trainor, L.J., 2006. One year of musical training affects development of auditory cortical-evoked fields in young children. Brain 129, 2593-2608.

Gilley, P.M., Sharma, A., Dorman, M., Martin, K., 2005. Developmental changes in refractoriness of the cortical auditory evoked potential. Clin Neurophysiol 116, 648-657.

Heim, S., Eulitz, C., Elbert, T., 2003. Altered hemispheric asymmetry of auditory P100m in dyslexia. European Journal of Neuroscience 17, 1715-1722.

Herrmann, B., Maess, B., Hahne, A., Schroger, E., Friederici, A.D., 2011. Syntactic and auditory spatial processing in the human temporal cortex: an MEG study. Neuroimage 57, 624-633.

Hertrich, I., Mathiak, K., Lutzenberger, W., Ackermann, H., 2000. Differential impact of periodic and aperiodic speech-like acoustic signals on magnetic M50/M100 fields. Neuroreport 11, 4017-4020.

Huotilainen, M., Shestakova, A., Hukki, J., 2008. Using magnetoencephalography in 
assessing auditory skills in infants and children. International Journal of Psychophysiology $68,123-129$.

Johnson, B.W., Crain, S., Thornton, R., Tesan, G., Reid, M., 2010. Measurement of brain function in pre-school children using a custom sized whole-head MEG sensor array. Clin Neurophysiol 121, 340-349.

Kajikawa, S., Amano, S., Kondo, T., 2004. Speech overlap in Japanese mother-child conversations. J Child Lang 31, 215-230.

Kaufman, A., Kaufman, N., 1983. Kaufman Assessment Battery for Children: Administration and Scoring Manual. Circle Pines MN: American Guidance Service.

Kotilahti, K., Nissila, I., Nasi, T., Lipiainen, L., Noponen, T., Merilainen, P., Huotilainen, M., Fellman, V., 2010. Hemodynamic responses to speech and music in newborn infants. Hum Brain Mapp 31, 595-603.

Leppänen, P.H.T., Hamalainen, J.A., Guttorm, T.K., Eklund, K.M., Salminen, H., Tanskanen, A., Torppa, M., Puolakanaho, A., Richardson, U., Pennala, R., Lyytinen, H., 2012. Infant brain responses associated with reading-related skills before school and at school age. Neurophysiologie Clinique-Clinical Neurophysiology 42, 35-41.

Leppänen, P.H.T., Hamalainen, J.A., Salminen, H.K., Eklund, K.M., Guttorm, T.K., Lohvansuu, K., Puolakanaho, A., Lyytinen, H., 2010. Newborn brain event-related 
potentials revealing atypical processing of sound frequency and the subsequent association with later literacy skills in children with familial dyslexia. Cortex 46, 1362-1376.

Oram Cardy, J.E., Ferrari, P., Flagg, E.J., Roberts, W., Roberts, T.P., 2004. Prominence of M50 auditory evoked response over M100 in childhood and autism. Neuroreport 15, 1867-1870.

Oram Cardy, J.E., Flagg, E.J., Roberts, W., Roberts, T.P., 2008. Auditory evoked fields predict language ability and impairment in children. Int J Psychophysiol 68, 170-175.

Orekhova, E.V., Butorina, A.V., Tsetlin, M.M., Novikova, S.I., Sokolov, P.A., Elam, M., Stroganova, T.A., 2013. Auditory magnetic response to clicks in children and adults: its components, hemispheric lateralization and repetition suppression effect. Brain topography $26,410-427$.

Orekhova, E.V., Tsetlin, M.M., Butorina, A.V., Novikova, S.I., Gratchev, V.V., Sokolov, P.A., Elam, M., Stroganova, T.A., 2012. Auditory Cortex Responses to Clicks and Sensory Modulation Difficulties in Children with Autism Spectrum Disorders (ASD). Plos One 7.

Pang, E.W., 2011. Practical aspects of running developmental studies in the MEG. Brain Topogr 24, 253-260.

Pang, E.W., Taylor, M.J., 2000. Tracking the development of the N1 from age 3 to adulthood: an examination of speech and non-speech stimuli. Clin Neurophysiol 111, 388-397. 
Pihko, E., Mickos, A., Kujala, T., Pihlgren, A., Westman, M., Alku, P., Byring, R., Korkman, M., 2007. Group intervention changes brain activity in bilingual language-impaired children. Cereb Cortex 17, 849-858.

Ponton, C., Eggermont, J.J., Khosla, D., Kwong, B., Don, M., 2002. Maturation of human central auditory system activity: separating auditory evoked potentials by dipole source modeling. Clin Neurophysiol 113, 407-420.

Sakai, K.L., 2005. Language acquisition and brain development. Science 310, 815-819.

Sharma, A., Kraus, N., McGee, T.J., Nicol, T.G., 1997. Developmental changes in P1 and N1 central auditory responses elicited by consonant-vowel syllables. Electroencephalogr Clin Neurophysiol 104, 540-545.

Squires, T., 2009. A discourse Anlysis of the Japanese Particle sa. Pragmatics 4, 1-29.

Sussman, E., Steinschneider, M., Gumenyuk, V., Grushko, J., Lawson, K., 2008. The maturation of human evoked brain potentials to sounds presented at different stimulus rates. Hearing Research 236, 61-79.

Tavabi, K., Obleser, J., Dobel, C., Pantev, C., 2007. Auditory evoked fields differentially encode speech features: an MEG investigation of the P50m and N100m time courses during syllable processing. Eur J Neurosci 25, 3155-3162.

Wilke, M., Holland, S.K., Altaye, M., Gaser, C., 2008. Template-O-Matic: A toolbox for 
creating customized pediatric templates. Neuroimage 41, 903-913.

Yoshimura, Y., Kikuchi, M., Shitamichi, K., Ueno, S., Munesue, T., Ono, Y., Tsubokawa, T., Haruta, Y., Oi, M., Niida, Y., Remijn, G.B., Takahashi, T., Suzuki, M., Higashida, H., Minabe, Y., 2013. Atypical brain lateralisation in the auditory cortex and language performance in 3to 7-year-old children with high-functioning autism spectrum disorder: a child-customised magnetoencephalography (MEG) study. Molecular autism 4, 38.

Yoshimura, Y., Kikuchi, M., Shitamichi, K., Ueno, S., Remijn, G.B., Haruta, Y., Oi, M., Munesue, T., Tsubokawa, T., Higashida, H., Minabe, Y., 2012. Language performance and auditory evoked fields in 2- to 5-year-old children. Eur J Neurosci 35, 644-650. 


\section{Figure Legends}

Figure 1. Auditory evoked fields and P1m component in one subject.

Figure 2. Trajectories of the P1m amplitude in the left (a) and right (b) hemispheres. In the left and right hemispheres, P1m was detected during both measurements (i.e., first and second measurements) in 17/20 and 18/20 participants, respectively. The bold red line is the regression line that corresponds to all data (i.e., the combination of the first and second measurements).

Figure 3. Trajectories of the P1m latency in the left (a) and right hemispheres (b). In the left and right hemispheres, P1m was detected for both measurements (i.e., first and second measurements) in $17 / 20$ and 18/20 participants, respectively. The bold red line is the regression line that corresponds to all data (i.e., the combination of the first and second measurements).

Figure 4. The correlation between the percent change in the standardized score of the language conceptual inference task in $\mathrm{K}-\mathrm{ABC}$ and the $\mathrm{P} 1 \mathrm{~m}$ amplitude change in the left 
(a) and right (b) hemispheres. In the left and right hemispheres, P1m was detected for both measurements (i.e., first and second measurements) in 17/20 and 18/20 participants, respectively.

Figure 5. The correlation between the percent change in the standardized score of the language conceptual inference task in $\mathrm{K}-\mathrm{ABC}$ and the change in $\mathrm{P} 1 \mathrm{~m}$ latency in the left (a) and right (b) hemispheres. In the left and right hemispheres, P1m was detected for both measurements (i.e., first and second measurements) in 17/20 and 18/20 participants, respectively. 
Table 1. Demographic characteristics of all participants.

\begin{tabular}{|c|c|c|c|c|}
\hline & First measurement & Second measurement & $\bar{t}$ & $P$ \\
\hline Number of subjects & \multicolumn{2}{|c|}{20} & & \\
\hline Gender (male/female) & \multicolumn{2}{|c|}{$17 / 3$} & & \\
\hline Chronological age (months) & $51.0( \pm 9.7)$ & $69.0( \pm 8.9)$ & 22.20 & $<.0001$ \\
\hline Head size $(\mathrm{cm})$ & $50.7( \pm 1.8)$ & $51.6( \pm 1.8)$ & 5.37 & $<.0001$ \\
\hline \multicolumn{5}{|l|}{$\mathrm{K}-\mathrm{ABC}$} \\
\hline Mental Processing Scale & $98.2( \pm 7.8)$ & $99.5( \pm 11.5)$ & 0.13 & n.s. \\
\hline Achievement Scale & $99.7( \pm 17.1)$ & $100.1( \pm 12.7)$ & 0.11 & n.s. \\
\hline Language Conceptual Inference Task (Riddles) & $96.6( \pm 17.2)$ & $97.2( \pm 12.9)$ & -0.19 & n.s. \\
\hline
\end{tabular}

K-ABC, Kaufman Assessment Battery for Children.

The values are the mean (and standard deviation) for chronological age, head size and standardized scores for the Mental

Processing Scale, Achievement Scale and Language Conceptual Inference task in the K-ABC. n.s., not significant 
Table 2. Repeated measures two-way ANOVA for the P1m amplitude and latency.

\begin{tabular}{|c|c|c|c|c|}
\hline & P1m amplitude & & P1m latency & \\
\hline & $F(1,15)$ & $P$ & $F(1,15)$ & $P$ \\
\hline Measurement time & 12.724 & 0.003 & 2.243 & n.s. \\
\hline Hemisphere & 18.935 & 0.001 & 4.192 & n.s. \\
\hline Interaction & 4.414 & n.s. & 0.466 & n.s. \\
\hline
\end{tabular}

Interaction: interaction between Measurement time and Hemisphere. n.s., not significant. 
Table 3. Location of the P1m source.

\begin{tabular}{ccc}
\hline & Left & Right \\
\hline EV in the first measurement & $0.047(1.11)$ & $-0.236(1.00)$ \\
EV in the second measurement & $-0.047(0.91)$ & $0.262(0.96)$ \\
\hline $\mathrm{N}$ & 0.31 & -3.58 \\
$P$ (first vs. second) & n.s. & 0.002
\end{tabular}

In both hemispheres, positive EV indicates locations toward the medial-vertex direction. EV, eigenvalue. 
Table 4. Values of the standardized regression coefficient $\beta$ and t values for the multiple linear regression models using the change in $\mathrm{P} 1 \mathrm{~m}$ amplitude as the dependent variable. The age at first measurement, time interval, percent change in the standardized score of the mental processing scale on the $\mathrm{K}-\mathrm{ABC}$ and percent change in the language conceptual inference task were independent variables.

\begin{tabular}{|c|c|c|c|c|}
\hline & \multicolumn{2}{|c|}{ Left hemisphere } & \multicolumn{2}{|c|}{ Right hemisphere } \\
\hline $\mathrm{N}$ & \multicolumn{2}{|c|}{17} & \multicolumn{2}{|c|}{18} \\
\hline \multirow[t]{2}{*}{$R^{2}$} & \multicolumn{2}{|c|}{$0.620 *$} & \multicolumn{2}{|c|}{0.260} \\
\hline & $\beta$ & $\mathrm{t}$ & $\beta$ & $\mathrm{t}$ \\
\hline Age (months) at first measurement & 0.027 & 0.139 & -0.301 & -1.109 \\
\hline Time interval & 0.476 & $2.457 *$ & 0.262 & 0.988 \\
\hline Change in mental processing scale & 0.277 & 1.524 & 0.199 & 0.832 \\
\hline Change in language conceptual inference task & 0.473 & $2.520 *$ & 0.033 & 0.135 \\
\hline
\end{tabular}




\section{Figure 1}

(a) First measurement

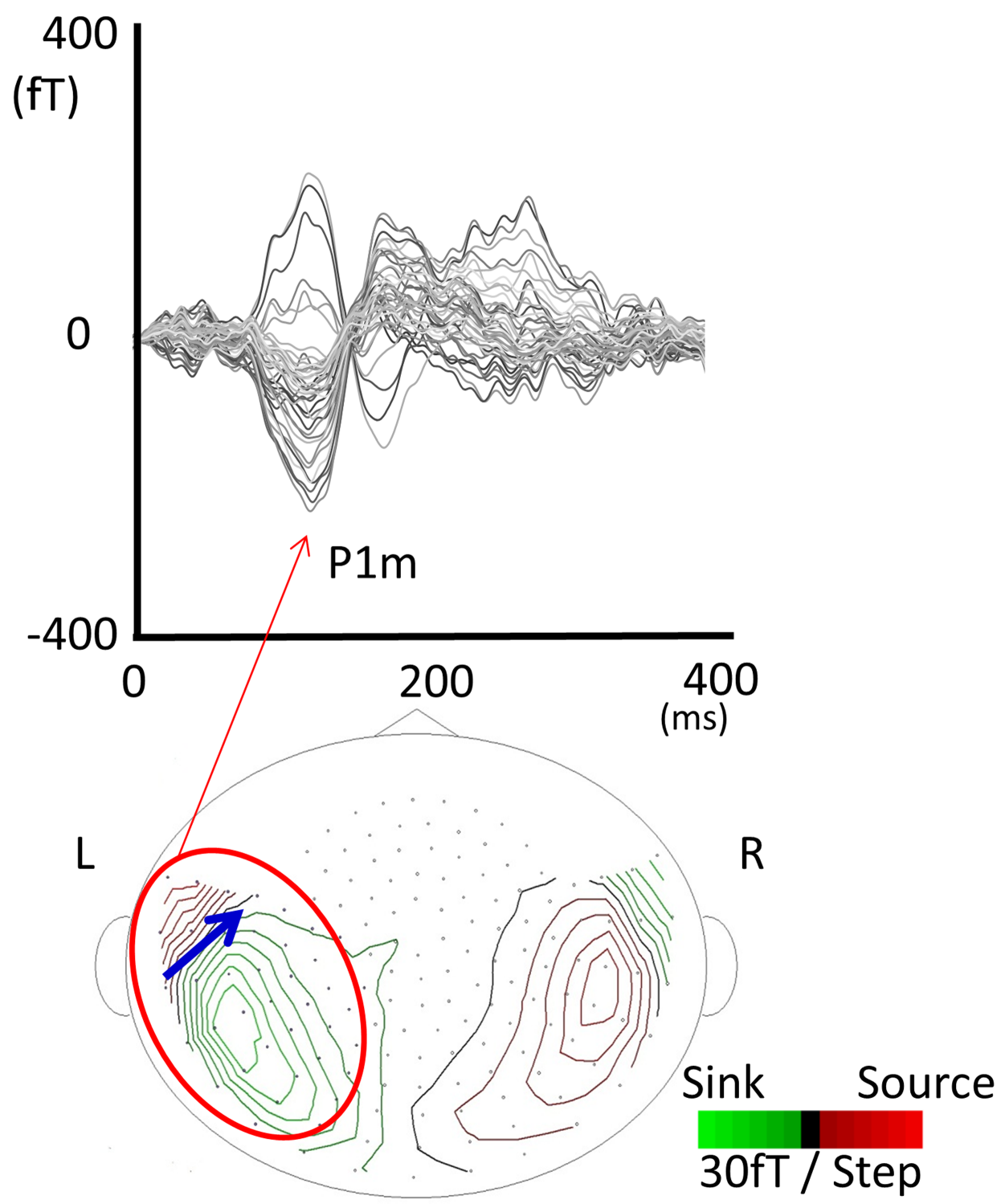

(b) Second measurement

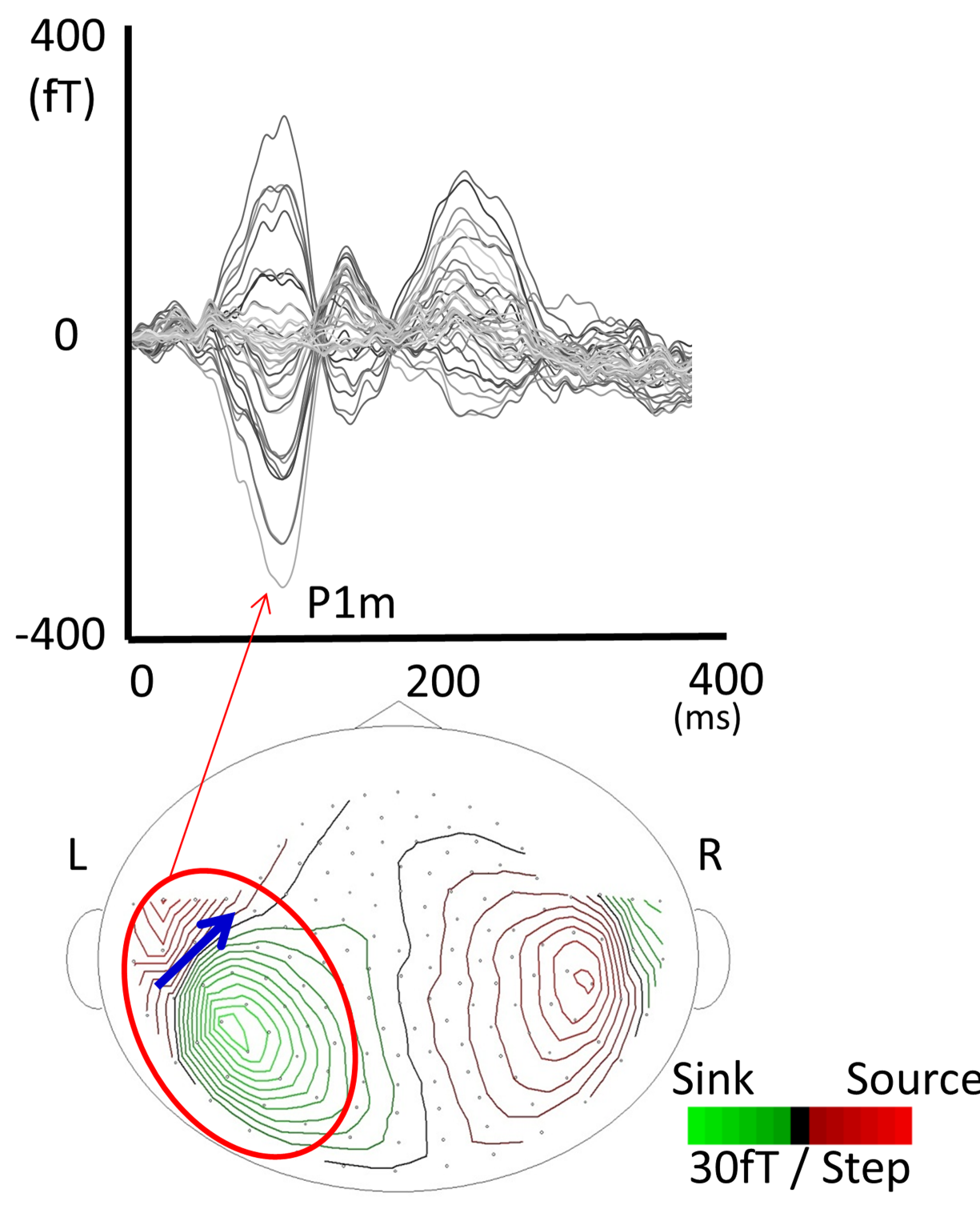




\section{Figure 2}

(a) Left hemisphere

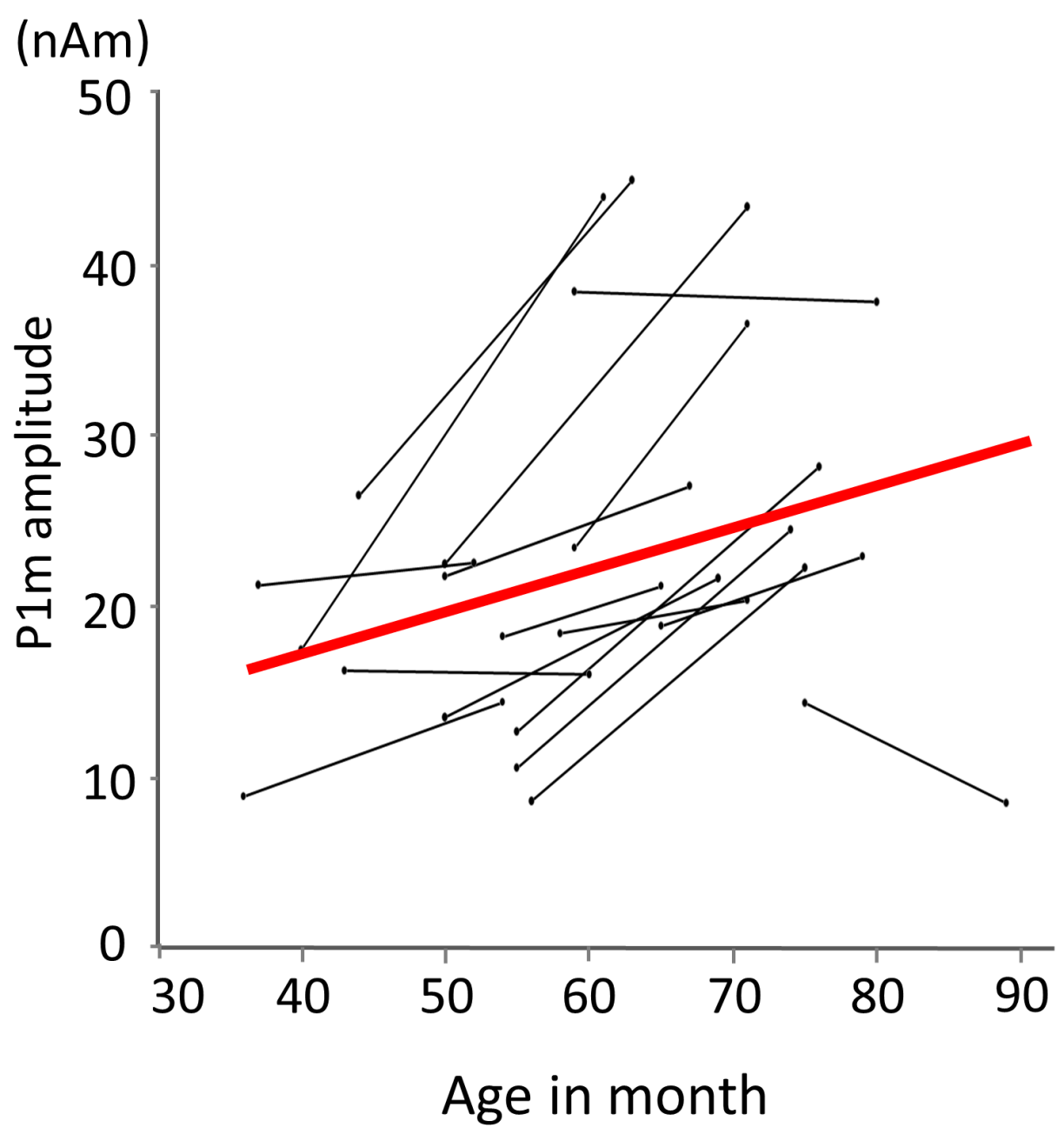

(b) Right hemisphere

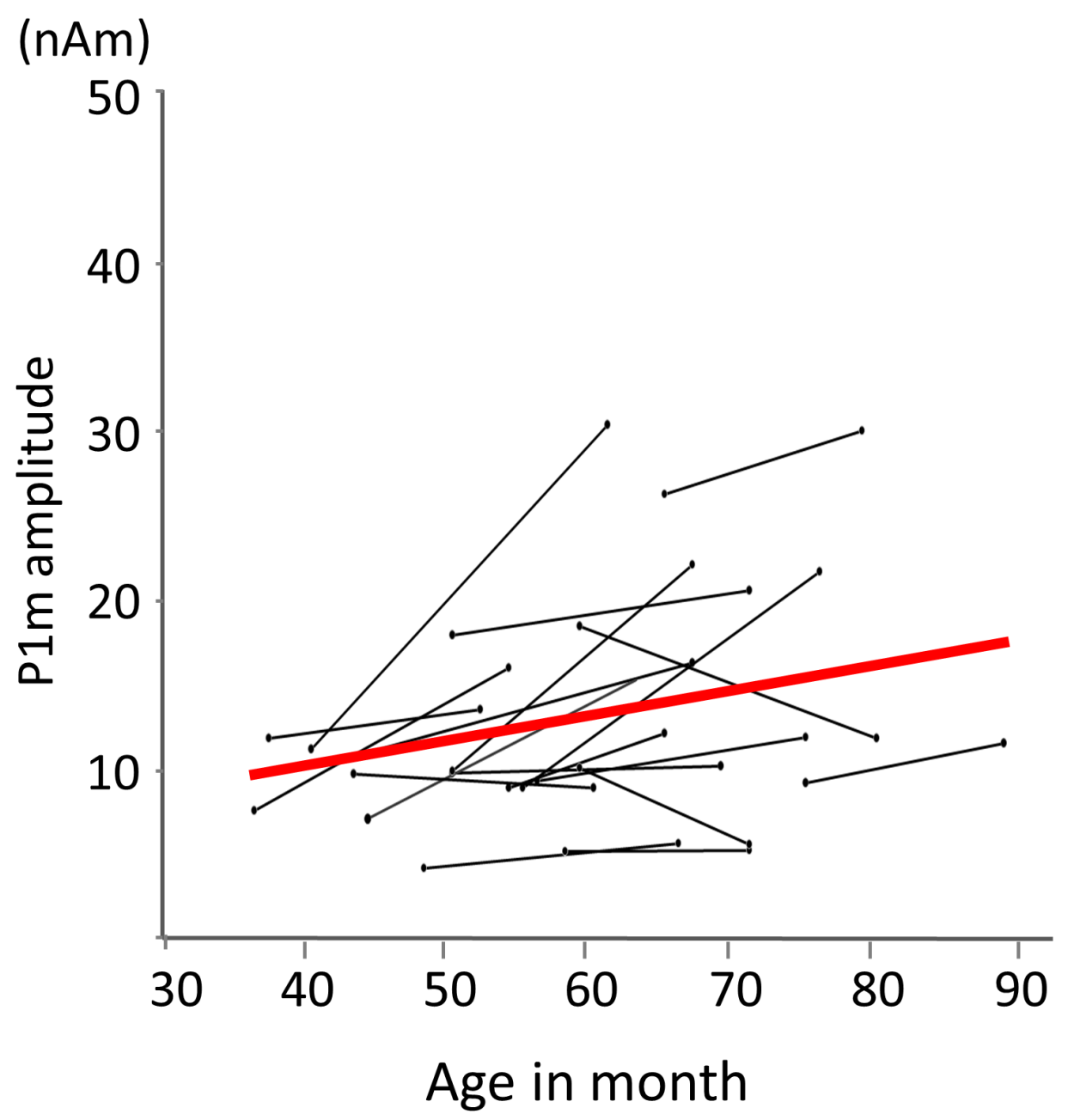


Figure 4

(a) Left hemisphere

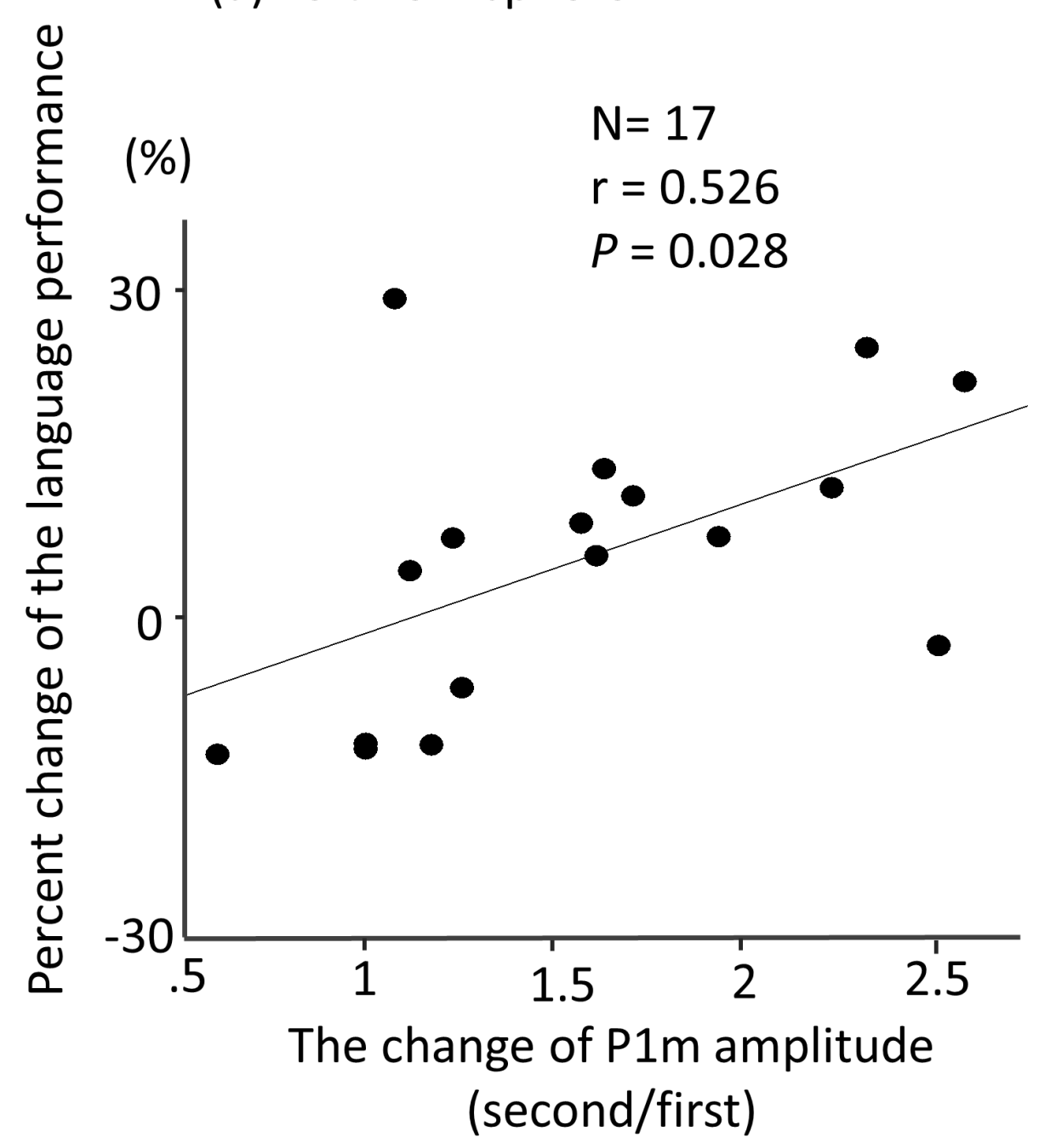

(b) Right Hemisphere

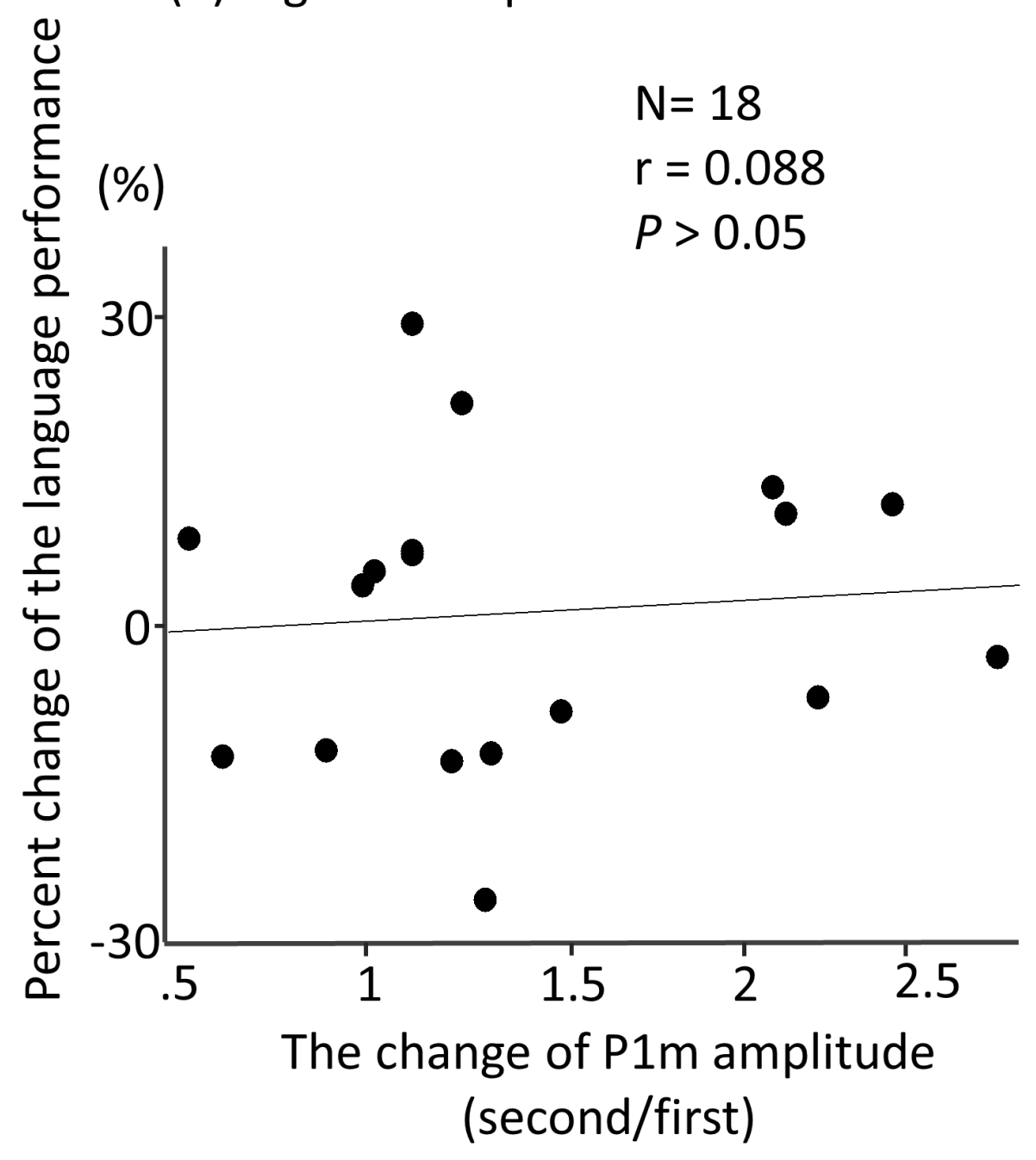


Figure 5
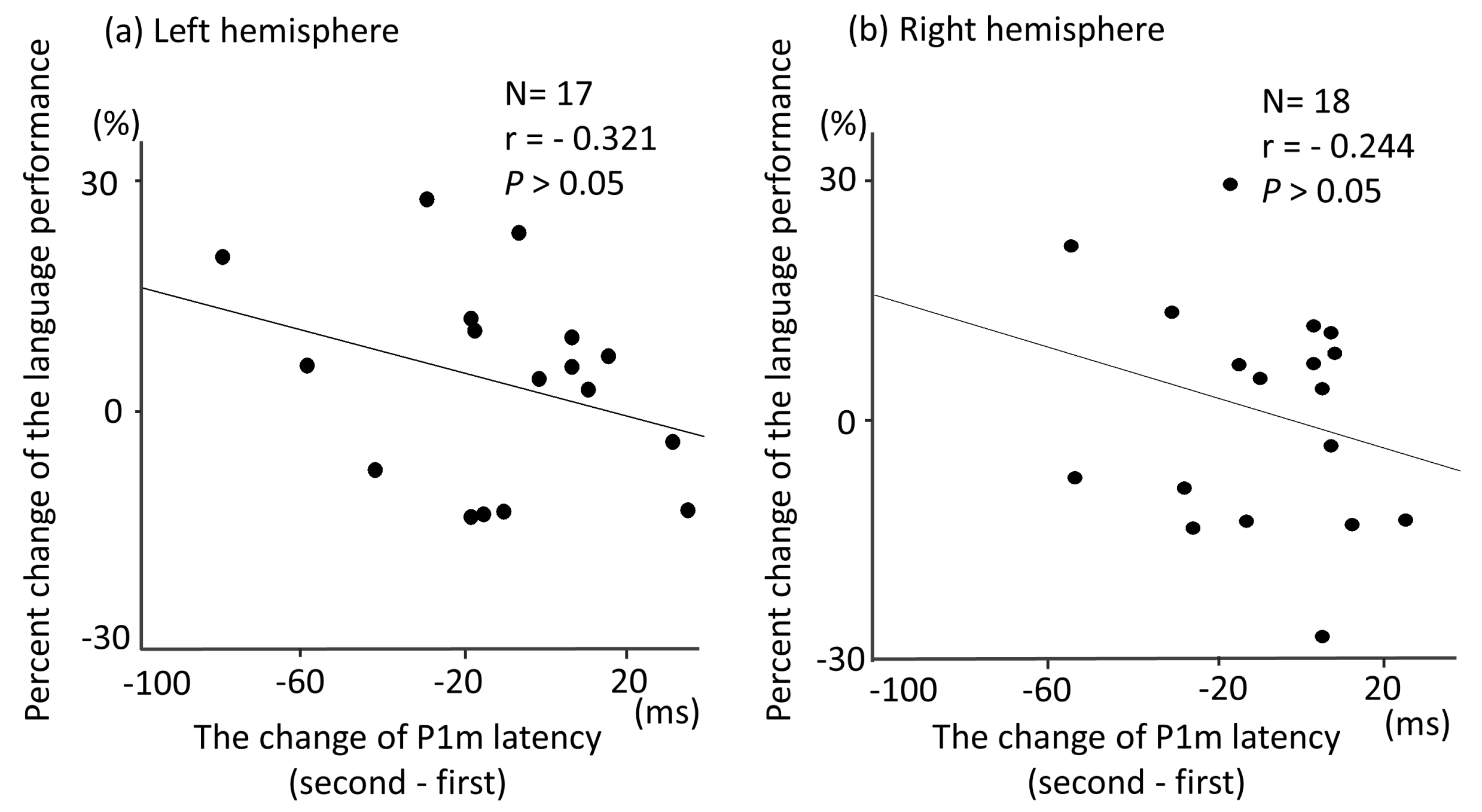